\title{
ENVELHECIMENTO ACELERADO EM SEMENTES DE Zinnia elegans Jacq. COLHIDAS EM DIFERENTES ÉPOCAS ${ }^{1}$
}

\author{
VANESSA OCOM MENEZES², DANIELE CARDOSO PEDROSO ${ }^{2}$, MARLOVE FÁTIMA BRIÃO MUNIZ \\ ROGÉRIO BELLÉ ${ }^{4}$, ELENA BLUME ${ }^{3}$, DANTON CAMACHO GARCIA ${ }^{4}$
}

\begin{abstract}
RESUMO - O objetivo do trabalho foi utilizar o teste de envelhecimento acelerado para avaliar o potencial fisiológico de sementes de Zinnia elegans Jacq., cultivar Gigante da Califórnia, colhidas em três diferentes épocas, bem como identificar os gêneros de fungos associados às sementes e sua influência sobre a qualidade das mesmas. Utilizaram-se sementes colhidas em janeiro, fevereiro e março de 2005, as quais foram submetidas ao envelhecimento acelerado em incubadora tipo BOD a $40^{\circ} \mathrm{C}$ por $0,24,48,72$ e 96 horas. A qualidade fisiológica foi avaliada pelos testes de germinação, primeira contagem, comprimento de plântula, emergência de plântulas em campo e a qualidade sanitária pelo "Blotter Test". O teste de envelhecimento acelerado conduzido à temperatura de $40^{\circ} \mathrm{C}$, por 72 e $96 \mathrm{~h}$, foi eficiente para diferenciação da qualidade das sementes de zínia, colhidas em diferentes épocas. $\mathrm{O}$ fungo Aspergillus spp., associado às sementes, influenciou negativamente na germinação.
\end{abstract}

Termos para indexação: zínia, vigor, fungos.

\section{ACCELERATED AGEING IN Zinnia elegans Jacq. SEEDS HARVESTED AT DIFFERENT TIMES IN DIFFERENT SEASONS}

\begin{abstract}
The objective of the work was to determine the time of exposure adequate to the assessment of physiological potential of the seeds of Zinnia elegans Jacq., cultivar "Single mixed", picked in three different epochs, through the accelerated ageing test, as well as identifying genera of fungi associated to the seeds and their influence on the quality of themselves. It was utilized seeds harvested in january, february and march of 2005, which remained in BOD chamber at $40^{\circ} \mathrm{C}$ for 0 , 24, 48, 72 and 96 hours. The physiological quality was evaluated by the tests of germination, first counting, the seedling length, rising of seedling, in field and plant length, and, the sanitary quality by "Blotter Test". The test of accelerated ageing was efficient for the stratification of the quality of the seeds of zinnia harvested in different seasons due to the physiological potential. The periods of the accelerated ageing of 72 and $96 \mathrm{~h}$, at temperature of $40^{\circ} \mathrm{C}$, were the ones that demonstrated greater capacity of seed quality physiological differentiation. The fungus Aspergillus spp. associated to the seeds negatively in the germination.
\end{abstract}

Index terms: zinnia, vigor, accelerated aging, fungi.

\footnotetext{
${ }^{1}$ Submetido em 18/10/2006. Aceito para publicação 15/09/2007.

${ }^{2}$ Bióloga, mestranda do PPG em Agronomia, Depto de Defesa Fitossanitária, Universidade Federal de Santa Maria (UFSM), vane_menezes@yahoo. com.br; dani.pedroso@yahoo.com.br
}

CEP: 97105-900. Santa Maria-RS, marlove@smail.ufsm.br; eblume@ smail.ufsm.br

${ }^{4} \mathrm{Eng}^{\mathrm{o}}$. Agr ${ }^{\mathrm{o}}$, Dr., Prof ${ }^{\mathrm{o}}$. Adjunto, Depto. de Fitotecnia, UFSM, CEP:97105900, Santa Maria-RS, belle@smail.com.br; danton@smail.ufsm.br.

${ }^{3}$ Enga. Agra, Dra., Profa. Adjunta, Depto. de Defesa Fitossanitária, UFSM, 


\section{INTRODUÇÃO}

Zinnia elegans é uma angiosperma da família Asteraceae, originária do México, conhecida popularmente como capitão, moça-e-velha, canela-de-velho ou zínia. É uma planta herbácea, anual, de caule ereto, folhagem áspera, flores pequenas reunidas em capítulos com uma vasta diversidade de cores e formas (Gemtchújnicov, 1976).

Devido à sua abundância à variedade de cores de suas flores, à grande diversidade de forma das brácteas (Torres, 1963) e à possibilidade de ser cultivada em qualquer época do ano (Lorenzi e Souza, 1999), zínia se destaca entre as espécies de maior potencial para cultivo como flor de corte em vasos e visando ornamentação. A produção e o consumo de flores e plantas ornamentais no Brasil vem se intensificando nos últimos anos e hoje, o país já se projeta no cenário internacional como importante referencial de qualidade e competitividade (Junqueira e Peetz, 2002).

A planta de zínia destaca-se, também, por apresentar um longo período de florescimento, devido ao desenvolvimento das gemas localizadas na base dos ramos. No entanto, esse longo período representa um obstáculo à obtenção de sementes de elevado vigor, pois, geralmente, apresentam baixo poder germinativo (Guimarães et al., 1998). Para obtenção de sementes com qualidade superior devem ser conhecidas as modificações que ocorrem nos teores de água, no conteúdo de matéria seca, na germinação e no vigor durante o seu desenvolvimento (Sader e Silveira, 1988), ou seja, definir a época de maturação fisiológica ideal para colheita das sementes. Além disso, em várias partes do mundo, zínia foi descrita como hospedeira natural ou experimental de patógenos (Maritan et al., 2004). Portanto, suas sementes merecem uma especial atenção no que se refere à avaliação de sua qualidade fisiológica e sanitária.

A qualidade da semente é o somatório de todos os atributos genéticos, físicos, fisiológicos e sanitários que afetam a sua capacidade de originar plantas de alta produtividade. A qualidade fisiológica da semente significa sua capacidade para desenvolver funções vitais, abrangendo germinação, vigor e longevidade (Popinigis, 1985). Já a qualidade sanitária das sementes é conseqüência da ação integrada de uma série de fatores, que ocorrem durante todo o processo de produção. É uma característica que deve ser avaliada, uma vez que a associação de patógenos às sementes pode implicar em redução do rendimento e comprometimento da qualidade das mesmas (Machado, 1988).

As sementes constituem-se em eficientes meios de disseminação e transmissão de patógenos e, freqüentemente, introduzem novos patógenos em áreas isentas. $\mathrm{O}$ inóculo inicial da epidemia pode depender da transmissão do patógeno pela semente e a presença do mesmo pode também, reduzir a qualidade fisiológica das sementes. A interação entre os testes de qualidade fisiológica e de sanidade de sementes, portanto, é de fundamental importância (Neegaard, 1979; Menten, 1995).

Entre os testes mais indicados para a avaliação da qualidade fisiológica de sementes está o teste de envelhecimento acelerado, o qual consiste em submeter às mesmas às condições adversas de alta temperatura (40$45^{\circ} \mathrm{C}$ ) e umidade relativa do ar de $100 \%$, durante certo período e, em seguida, observar a resposta através do teste padrão de germinação (Marcos Filho, 1999). Nessa situação, sementes que apresentam menor qualidade deterioram-se mais rapidamente do que as mais vigorosas, com reflexos na germinação após o período de envelhecimento artificial (Miranda et al., 2001). A eficiência desse teste é avaliada pela diferença de sensibilidade apresentada pelas sementes ao envelhecimento.

Para algumas espécies, como soja, milho e trigo, estudos de envelhecimento acelerado já estão padronizados, ou seja, indicam a temperatura e o período ideal à realização desse teste, de modo a classificar as sementes quanto ao seu vigor. Entretanto, para espécies ornamentais, como zínia, o uso do teste ainda é limitado.

Portanto, realizou-se este trabalho com o objetivo de utilizar o teste de envelhecimento acelerado para a avaliação do potencial fisiológico de sementes de Zinnia elegans, colhidas em três diferentes épocas, bem como identificar os gêneros de fungos associados às sementes e sua influência sobre a qualidade das mesmas.

\section{MATERIAL E MÉTODOS}

O trabalho foi conduzido no laboratório de Fitopatologia do Departamento de Defesa Fitossanitária da Universidade Federal de Santa Maria (UFSM), RS. Foram utilizadas sementes de Zinnia elegans Jacq., da cultivar Gigante da Califórnia, provenientes da área experimental do Departamento de Fitotecnia (UFSM), colhidas manualmente, em três épocas: 17 de janeiro, 10 de fevereiro e 15 de março do ano de 2005. Os capítulos colhidos em janeiro e março tinham aparência seca e aqueles colhidos em fevereiro apresentavam-se verdolengos.

Para a avaliação da qualidade fisiológica, as sementes obtidas em cada época de colheita foram submetidas às condições de envelhecimento acelerado, utilizando-se 
caixas plásticas transparentes $(11,5 \times 11,5 \times 3,5 \mathrm{~cm})$ como compartimentos individuais, no lugar das telas metálicas tradicionais, foram utilizadas gazes para dar suporte às sementes. Na superfície destas gazes, foram distribuídas, em camada única, aproximadamente $5 \mathrm{~g}$ de sementes de cada época de colheita. Para a obtenção de umidade relativa do ar de $100 \%$ no interior das caixas, foram colocados $40 \mathrm{~mL}$ de água. As caixas tampadas foram mantidas em incubadora do tipo "BOD" regulada a $40^{\circ} \mathrm{C}$, por períodos de $0,24,48$, 72 e 96 horas. Decorridos os períodos as sementes foram submetidas aos testes descritos a seguir: grau de umidade - para cada período de envelhecimento quatro subamostras foram retiradas, com $5 \mathrm{~g}$ de sementes para a avaliação do grau de umidade, realizado pelo método da estufa a $105 \pm 3{ }^{\circ} \mathrm{C}$ por 24 horas (Brasil, 1992); germinação - conduzido com oito repetições de 25 sementes distribuídas sobre duas folhas de papel filtro umedecido com água destilada, em caixas plásticas tipo gerbox, mantidas em câmara climatizada, a $25^{\circ} \mathrm{C}$, com fotoperíodo de 12 horas e as avaliações realizadas aos cinco e dez dias após a semeadura (Brasil, 1992). Os resultados foram expressos em porcentagem de plântulas normais, avaliando-se, também, a porcentagem de sementes mortas; primeira contagem de germinação - conduzido junto com o teste de germinação, constituiu no registro das porcentagens de plântulas normais verificadas na primeira contagem da germinação (quinto dia); comprimento de plântulas - foram medidos os comprimentos totais das plântulas, determinados em centímetros, com o auxílio de uma régua milimetrada, no quinto dia após a semeadura. Calculou-se o comprimento médio por plântula ( $\mathrm{cm} /$ plântula), dividindo o somatório dos valores obtidos pelo número de plântulas normais da primeira contagem da germinação; classificação do vigor de plântulas - foi conduzido conjuntamente com o teste de germinação, consistindo no registro da porcentagem de plântulas normais "fortes" verificadas na primeira contagem de germinação. Sendo consideradas normais "fortes" aquelas que se apresentavam sadias, com cotilédones e sistema radicular bem desenvolvido, conforme recomendou Nakagawa, 1999; emergência de plântulas em campo - conduzido em casa de vegetação, utilizando-se bandejas contendo substrato comercial, realizado com quatro repetições de dez sementes para cada época de colheita. Foram feitas irrigações sempre que necessário e a avaliação foi realizada aos trinta dias após a semeadura, computando-se a porcentagem de plântulas emersas; determinou-se ainda o comprimento total da plântula, em cm/plântula; comprimento de plântula realizado em conjunto com o teste de emergência em campo, avaliando-se o comprimento médio das plantas obtidas aos trinta dias após a semeadura, determinados em centímetros, com o auxílio de uma régua milimetrada. Calculou-se o comprimento médio por plântula ( $\mathrm{cm} /$ plântula), dividindo o somatório dos valores obtidos pelo número de plântulas normais.

A avaliação sanitária das sementes foi realizada pelo "Blotter Test", com oito repetições de 25 sementes. As sementes foram distribuídas em caixas "gerbox", utilizandose duas folhas de papel filtro umedecidas com água estéril na proporção de 2,5 vezes o peso do papel. As caixas foram mantidas em câmara de crescimento, a $25^{\circ} \mathrm{C}$, em regime alternado de 12 horas de luz por sete dias. Após o período de incubação, as sementes foram examinadas individualmente, com auxílio de lupa estereoscópica e microscópio óptico, para verificação da presença de patógenos, os quais foram identificados no nível de gênero, através das suas estruturas morfológicas, determinando-se a porcentagem de sementes infestadas por fungos. Essa identificação, também, foi baseada na bibliografia especializada de Barnett e Hunter (1998).

O delineamento experimental empregado foi $o$ completamente casualizado. Os dados foram submetidos à análise de variância e ao teste $\mathrm{F}$, utilizando esquema fatorial $4 \times 3$ (quatro tempos de exposição ao envelhecimento e três épocas de colheita), as variáveis, germinação, sementes mortas e incidência de fungos foram analisadas separadamente através da análise de correlação, empregando-se o Programa de Análise Estatística SANEST (Zonta e Machado, 1986). A comparação das médias foi realizada pelo teste de Tukey a $5 \%$ de probabilidade.

\section{RESULTADOS E DISCUSSÃO}

Na Tabela 1 encontram-se os resultados referentes ao grau de umidade das sementes de Zinnia elegans Jacq. antes e após o de envelhecimento acelerado. Observou-se uma variação em relação ao grau de umidade inicial das sementes para cada mês de colheita, aumentando conforme o tempo de exposição ao teste de envelhecimento acelerado. No mês de janeiro, as sementes apresentaram grau de umidade inicial de $18,82 \%$, após $24,48,72$ e $96 \mathrm{~h}$ de envelhecimento acelerado, esse grau de umidade aumentou para 31,99\%, 32,93\%, $33,87 \%$ e $34,81 \%$ respectivamente. No mês de fevereiro, o grau de umidade das sementes aumentou de $30,84 \%$ para $52,59 \%, 54,14 \%, 55,69 \%$ e $57,23 \%$ com $24,48,72$ e $96 \mathrm{~h}$ respectivamente. Em março, o grau de umidade das sementes no tempo zero de exposição foi de $14,82 \%$, ocorrendo elevação para $25,19 \%, 25,93 \%, 26,67 \%$ e $27,41 \%$, nos 
tempos de 24, 48, 72 e 96h respectivamente. Essa diferença interferiu nos resultados obtidos nos testes que avaliaram a qualidade fisiológica das sementes, pois sementes com maior grau de umidade apresentaram menor vigor em relação às demais, principalmente no mês de fevereiro.

TABELA 1. Grau de umidade (\%) inicial e após o teste de envelhecimento acelerado de sementes de Zinnia elegans Jacq. colhidas em diferentes épocas. Santa Maria - RS, 2006.

\begin{tabular}{cccccc}
\hline \multirow{2}{*}{ Mês de Colheita } & \multicolumn{5}{c}{ Grau de Umidade (\%) } \\
\cline { 2 - 6 } & Inicial & $24 \mathrm{~h}$ & $48 \mathrm{~h}$ & $96 \mathrm{~h}$ \\
\hline Janeiro & 18,82 & 31,99 & 32,93 & 34,81 \\
Fevereiro & 30,84 & 52,59 & 54,14 & 33,87 & 57,23 \\
Março & 14.82 & 25,19 & 25,93 & 55,69 & 27,41 \\
\hline
\end{tabular}

Os dados de temperatura média, umidade relativa do ar e precipitação pluviométrica, referentes ao período do trabalho foram coletados na estação meteorológica da UFSM e se encontram na Tabela 2. É possível verificar, que no mês de fevereiro, obteve-se a mais alta porcentagem de umidade relativa e o maior índice de precipitação em relação aos demais meses, o que justifica a alta umidade das sementes colhidas nesse mês. Segundo Lopes et al (2005), a época ideal para a colheita de sementes pode ser afetada pelas condições ambientais.

TABELA 2. Médias de temperatura $\left({ }^{\circ} \mathrm{C}\right)$, umidade relativa $(\%)$ e precipitação $(\mathrm{mm})$ registradas durante os meses de colheita das sementes de Zinnia elegans Jacq. em 2005. Santa Maria-RS, 2006.

\begin{tabular}{cccc}
\hline Meses de colheita & Temperatura $\left({ }^{\circ} \mathrm{C}\right)$ & Umidade Relativa (\%) & Precipitação $(\mathrm{mm})$ \\
\hline Janeiro & 25,9 & $64 \%$ & 49,1 \\
Fevereiro & 24,3 & $71 \%$ & 59,4 \\
Março & 23,7 & $68 \%$ & 55,4 \\
\hline
\end{tabular}

Os resultados dos testes de germinação, primeira contagem de germinação, comprimento de plântula, classificação do vigor de plântulas e da variável, sementes mortas encontram-se na Tabela 3. No teste germinação, os períodos de exposição ao envelhecimento acelerado de 24, 72 e $96 \mathrm{~h}$ se mostraram promissores para uma estratificação da qualidade fisiológica das sementes em inferior, intermediária e superior, conforme o mês de colheita. Para o teste de primeira contagem de germinação, somente os tempos de 72 e $96 \mathrm{~h}$ se mostraram adequados para essa classificação de vigor, sendo que o período de $24 \mathrm{~h}$ estratificou as sementes apenas em dois níveis de qualidade. Resultados semelhantes foram observados por Nascimento et al (1993) em seus trabalhos com envelhecimento acelerado em sementes de tomate utilizando $42^{\circ} \mathrm{C} / 72 \mathrm{~h}$, onde esse teste apresentou sensibilidade suficiente para a estratificação de lotes em diferentes potenciais fisiológicos.

As sementes colhidas em fevereiro demonstraram um baixo potencial de germinação e de vigor no teste de primeira contagem. A qualidade inferior dessas sementes, provavelmente, se deve ao seu elevado grau de umidade, esse fato aliado à temperatura elevada $\left(40^{\circ} \mathrm{C}\right)$, resultou em um processo de deterioração acelerado. Segundo Carvalho e Nakagawa (2000), incrementos no grau de umidade favorecem a elevação da temperatura da semente, devido a processos respiratórios e de uma maior atividade de microrganismos, prejudicando assim, sua viabilidade.

$\mathrm{Na}$ avaliação do comprimento de plântula (Tabela $3)$, verifica-se que no tempo zero, ocorreu uma diferença significativa entre os meses de colheita, permitindo destacar o mês de março como o de potencial fisiológico superior, janeiro como intermediário e o de fevereiro como inferior. Entretanto, em $96 \mathrm{~h}$ de envelhecimento acelerado a estratificação observada permitiu classificar sementes colhidas no mês de janeiro como as mais vigorosas, ou seja, as que resultaram em plântulas com maiores médias de comprimento em relação às demais. 
TABELA 3. Médias obtidas nos testes utilizados para avaliação da qualidade fisiológica de sementes de Zinnia elegans Jacq., colhidas em janeiro (J), fevereiro (F) e março (M) de 2005, após serem submetidas a diferentes períodos de envelhecimento acelerado, Santa Maria-RS, 2006.

\begin{tabular}{|c|c|c|c|c|c|c|c|c|}
\hline \multirow{2}{*}{ Períodos (h) } & \multicolumn{4}{|c|}{ Germinação (\%) } & \multicolumn{4}{|c|}{ Primeira Contagem de Germinação (\%) } \\
\hline & $\mathrm{J}$ & $\mathrm{F}$ & $\mathrm{M}$ & Média & $\mathrm{J}$ & $\mathrm{F}$ & $\mathrm{M}$ & Média \\
\hline 0 & 78 a A & $17 \mathrm{~b} \mathrm{~A}$ & 77 a A & 58 & 64 a A & $3 \mathrm{~b} \mathrm{~A} \mathrm{~B}$ & 71 a $A$ & 42 \\
\hline 24 & 63 a A B & $7 \mathrm{c} \mathrm{A}$ & $44 \mathrm{~b} \mathrm{~B}$ & 36 & 54 a $\mathrm{AB}$ & $4 \mathrm{~b} \mathrm{~A} \mathrm{~B}$ & 43 a B & 30 \\
\hline 48 & 66 a A B & $12 \mathrm{~b} \mathrm{~A}$ & 52 a B & 42 & 60 a A & $6 \mathrm{~b} \mathrm{~A}$ & 50 a B & 35 \\
\hline 72 & 63 a B & $1 \mathrm{c} \mathrm{B}$ & $10 \mathrm{~b} \mathrm{C}$ & 19 & 40 a B & $1 \mathrm{c} \mathrm{B}$ & $13 \mathrm{~b} \mathrm{C}$ & 13 \\
\hline 96 & 44 a C & $1 \mathrm{c} \mathrm{B}$ & $10 \mathrm{~b} \mathrm{C}$ & 13 & 41 a B & $1 \mathrm{c} \mathrm{B}$ & $10 \mathrm{~b} \mathrm{C}$ & 12 \\
\hline Média & 63 & 6 & 38 & & 52 & 2 & 35 & \\
\hline C.V. (\%) & & 19,8 & & & & 25,08 & & \\
\hline \multirow{2}{*}{ Períodos (h) } & \multicolumn{4}{|c|}{ Comprimento de Plântulas $(\mathrm{cm})$} & \multicolumn{4}{|c|}{ Plântulas Normais Fortes (\%) } \\
\hline & $\mathrm{J}$ & $\mathrm{F}$ & M & Média & $\mathrm{J}$ & $\mathrm{F}$ & $\mathrm{M}$ & Média \\
\hline 0 & $4 \mathrm{~b}$ A B & $2 \mathrm{c} \mathrm{A}$ & 5 a A & 7 & 43 a $\mathrm{A}$ & $2 \mathrm{~b} \mathrm{~A}$ & 53 a A & 28 \\
\hline 24 & 5 a A & $2 \mathrm{~b} \mathrm{~A}$ & 5 a A & 4 & 22 a B & $1 \mathrm{~b} \mathrm{~A}$ & 17 a B & 10 \\
\hline 48 & 3 a B & $2 \mathrm{~b} \mathrm{~A}$ & 4 a A & 3 & 25 a B & $1 \mathrm{~b} \mathrm{~A}$ & 20 a B & 12 \\
\hline 72 & 3 a B & $1 \mathrm{~b} \mathrm{~B}$ & 3 a B & 2 & 12 a B & $0 \mathrm{~b} \mathrm{~A}$ & $2 \mathrm{~b} \mathrm{C}$ & 3 \\
\hline 96 & 4 a $A B$ & $1 \mathrm{c} \mathrm{B}$ & $2 \mathrm{~b} \mathrm{~B}$ & 2 & 13 a B & $0 \mathrm{c} \mathrm{A}$ & $5 \mathrm{~b} \mathrm{C}$ & 4 \\
\hline Média & 4 & 1 & 4 & & 23 & 1 & 16 & \\
\hline C.V. $(\%)$ & & 30,52 & & & & 37,76 & & \\
\hline \multirow{2}{*}{ Períodos (h) } & \multicolumn{4}{|c|}{ Sementes Mortas (\%) } & & & & \\
\hline & $\mathrm{J}$ & $\mathrm{F}$ & M & Média & & & & \\
\hline 0 & $5 \mathrm{~b} \mathrm{~B}$ & 43 a D & $12 \mathrm{~b} \mathrm{~B}$ & 17 & & & & \\
\hline 24 & $18 \mathrm{~b} \mathrm{~A}$ & 63 a C & $16 \mathrm{~b} \mathrm{~B}$ & 31 & & & & \\
\hline 48 & $18 \mathrm{c} \mathrm{A}$ & 67 a B C & $23 \mathrm{~b} \mathrm{~B}$ & 35 & & & & \\
\hline 72 & $25 \mathrm{c} \mathrm{A}$ & 80 a $\mathrm{A}$ B & $66 \mathrm{~b} \mathrm{~A}$ & 58 & & & & \\
\hline 96 & 30 c A & 82 a $\mathrm{A}$ & 61 & 58 & & & & \\
\hline \multicolumn{9}{|l|}{ Média } \\
\hline C.V. $(\%)$ & & 8,83 & & & & & & \\
\hline
\end{tabular}

*Médias seguidas pela mesma letra maiúscula na coluna e minúscula na linha não diferem entre si pelo teste de Tukey, em nível de 5\% de probabilidade.

No teste de classificação de vigor de plântulas (Tabela 3), apenas o tempo de envelhecimento acelerado de $96 \mathrm{~h}$ foi capaz de estratificar os meses de colheita das sementes de zínia, de acordo com seu vigor, da mesma forma que ocorreu no teste de comprimento de plântulas. Os demais tempos de exposição permitiram a classificação em apenas dois níveis de qualidade fisiológica.
Com relação à variável, sementes mortas, os resultados indicam que os períodos a partir de $48 \mathrm{~h}$ de envelhecimento acelerado foram significativos para provocar um aumento no número de sementes mortas, em relação ao tempo inicial. Do mesmo modo, os períodos de envelhecimento acelerado demonstraram sensibilidade para indicar que para o mês de fevereiro apresentou uma alta incidência de sementes 
mortas, o que pode ser devido ao alto grau de umidade das mesmas. À medida que essas sementes foram expostas a uma condição de umidade relativa de $100 \%$, por um tempo cada vez maior, seu grau de umidade aumentou, causando conseqüente deterioração das mesmas, devido ao propício desenvolvimento de fungos associados a essas sementes. Os meses de colheita de março e janeiro foram classificados como intermediário e inferior, respectivamente, em relação à porcentagem de sementes mortas.

Portanto, os maiores tempos de exposição às condições adversas proporcionadas pelo teste de envelhecimento acelerado, 72 e 96h, foram os que demonstraram maior capacidade de diferenciação fisiológica de qualidade de sementes de zínia colhidas em diferentes épocas. A partir dos testes realizados, sementes colhidas no mês de janeiro têm uma qualidade fisiológica superior em relação às demais, seguidas pelas de março e fevereiro. Esse resultado provavelmente ocorreu devido à desuniformidade das épocas de maturação, pois uma colheita precoce resulta em decréscimo na produção pelo desenvolvimento incompleto das sementes, que se apresentam com um alto conteúdo de umidade (Smiderle e Pereira, 2008), como se pode observar naquelas colhidas no mês de fevereiro.

Verifica-se, pela emergência de plântulas em campo (Tabela 4), que todos os períodos de exposição à condição artificial de envelhecimento acelerado foram eficientes para estratificar as sementes de zínia em três categorias fisiológicas. Para sementes de abobrinha, o teste de emergência realizado em areia avaliado aos 15 dias após a semeadura (DAS), mostrou-se apropriado na estratificação dos lotes em função do potencial fisiológico, obtendo correlação significativa com os testes de germinação e envelhecimento acelerado (Barros et al., 2002). Cardoso (2003) encontrou resultados semelhantes, para essa espécie, porém, as avaliações foram feitas aos 19 DAS. Já, no teste de comprimento de plântulas nenhum dos tempos de exposição ao envelhecimento acelerado foi capaz de estabelecer uma diferença estatística entre os meses de colheita das sementes.

TABELA 4. Médias obtidas nos testes de emergência e comprimento de plântulas de Zinnia elegans Jacq., obtidas de sementes colhidas em janeiro $(J)$, fevereiro $(F)$ e março $(M)$ de 2005, e submetidas a diferentes períodos (P) de envelhecimento acelerado, Santa Maria-RS, 2006.

\begin{tabular}{|c|c|c|c|c|c|c|c|c|}
\hline \multicolumn{5}{|c|}{ Emergência (\%) } & \multicolumn{4}{|c|}{ Comprimento de Planta (\%) } \\
\hline \multicolumn{5}{|c|}{ Meses de Colheita } & \multicolumn{4}{|c|}{ Meses de Colheita } \\
\hline $\mathrm{P}(\mathrm{h})$ & $\mathrm{J}$ & $\mathrm{F}$ & M & Média & $\mathrm{J}$ & $\mathrm{F}$ & $\mathrm{M}$ & Média \\
\hline 0 & $87 \mathrm{~b} \mathrm{~A}$ & $57 \mathrm{c} \mathrm{A}$ & 100 a $\mathrm{A}$ & 87 & $7 \mathrm{ab} \mathrm{A}$ & $5 \mathrm{~b} \mathrm{~A} \mathrm{~B}$ & 10 a $\mathrm{A}$ & 7 \\
\hline 24 & 62 a B C & $6 \mathrm{c} \mathrm{C}$ & 43 b B & 34 & 6 a A & 4 a A B & 5 a B & 5 \\
\hline 48 & 67 a B & $12 \mathrm{c} \mathrm{B}$ & $50 \mathrm{~b} \mathrm{~B}$ & 41 & 5 a $A$ & 8 a $A$ & 4 a B & 6 \\
\hline 72 & 56 a C & $1 \mathrm{c} \mathrm{D}$ & $10 \mathrm{~b} \mathrm{C}$ & 17 & 4 a A & 2 a B & 3 a B & 3 \\
\hline 96 & 42 a D & $2 \mathrm{c} \mathrm{D}$ & $8 \mathrm{~b} \mathrm{C}$ & 13 & 3 a A & 2 a B & 3 a B & 2 \\
\hline Média & 63 & 11 & 45 & & 5 & 4 & 5 & \\
\hline C.V. (\%) & \multicolumn{4}{|c|}{11,28} & \multicolumn{4}{|c|}{64,7} \\
\hline
\end{tabular}

* Médias seguidas pela mesma letra maiúscula na coluna e minúscula na linha não diferem entre si pelo teste de Tukey, ao nível de $5 \%$ de probabilidade. h: tempo de exposição em horas.

Os dados referentes à avaliação da qualidade sanitária de sementes de zínia submetidas a diferentes tempos de envelhecimento acelerado encontram-se na Tabela 5. Foram detectados os seguintes fungos associados às sementes: Aspergillus spp., Curvularia spp.,Fusarium spp. e Penicillium spp., com maior incidência e, também, Drechslera spp. e Alternaria spp., porém em baixos percentuais.

Os fungos Penicillium spp. e Aspergillus spp. destacaramse pela sua alta ocorrência, principalmente nas sementes colhidas em fevereiro, sendo que maiores tempos de envelhecimento (72 e 96h) resultaram em maior porcentagem de sementes associadas com Aspergillus spp.. Provavelmente, esses altos índices devam-se às características iniciais das sementes, como seu elevado grau de umidade, adicionada de uma maior quantidade de umidade, proporcionada pelo envelhecimento acelerado. Segundo Neergaard (1979), a maioria dos gêneros de fungos necessita de alta temperatura e umidade para seu melhor desenvolvimento. Choudhury 
(1987) afirmou que os danos causados pelas espécies de Aspergillus e de Penicillium são variáveis, como perda de germinação, descoloração das sementes, aumento da taxa de ácidos graxos, aquecimento da massa de sementes e produção de toxinas. Estudos realizados por Halloin (1975) mostraram que toxinas ou enzimas autocatalíticas podem estar envolvidas na deterioração das sementes, causada por Aspergillus spp.

TABELA 5. Porcentagem média de fungos associados às sementes de Zinnia elegans Jacq. colhidas em diferentes meses e expostas a diferentes períodos de envelhecimento acelerado. Santa Maria - RS, 2006.

\begin{tabular}{|c|c|c|c|c|c|c|c|c|c|c|c|c|}
\hline \multirow[b]{2}{*}{$\mathrm{P}(\mathrm{h})$} & \multicolumn{4}{|c|}{ Alternaria spp. } & \multicolumn{4}{|c|}{ Aspergillus spp. } & \multicolumn{4}{|c|}{ Penicillium spp. } \\
\hline & $\mathrm{J}$ & $\mathrm{F}$ & $\mathrm{M}$ & Média & $\mathrm{J}$ & $\mathrm{F}$ & $\mathrm{M}$ & Média & $\mathrm{J}$ & $\mathrm{F}$ & M & Média \\
\hline 24 & $10 \mathrm{Ba}^{*}$ & $2 \mathrm{Ca} \quad 1$ & $17 \mathrm{Aa}$ & 10 & $0 \mathrm{Bc}$ & $30 \mathrm{Ab}$ & $5 \mathrm{Bc}$ & 11 & $3 \mathrm{Bc}$ & $37 \mathrm{Ab}$ & $6 \mathrm{Ba}$ & 15 \\
\hline 48 & $4 \mathrm{Ab}$ & 0 Aa & $3 \mathrm{Ab}$ & 2 & $3 \mathrm{Bc}$ & $8 \mathrm{Bc}$ & $30 \mathrm{Ab}$ & b $\quad 14$ & $13 \mathrm{Bcb}$ & $85 \mathrm{Aa}$ & $24 \mathrm{Ba}$ & 39 \\
\hline 72 & $2 \mathrm{Ab}$ & 0 Aa & $0 \mathrm{Ab}$ & 0,8 & $43 \mathrm{Ba}$ & $89 \mathrm{Aa}$ & $75 \mathrm{Aa}$ & a 68 & $32 \mathrm{Ba}$ & $80 \mathrm{Aa}$ & $9 \mathrm{Ca}$ & 39 \\
\hline 96 & $2 \mathrm{Ab}$ & $0 \mathrm{Aa}$ & $0 \mathrm{Ab}$ & 0,8 & $28 \mathrm{Bb}$ & $93 \mathrm{Aa}$ & $87 \mathrm{Aa}$ & a 68 & $31 \mathrm{Bab}$ & $67 \mathrm{Aa}$ & $8 \mathrm{Ca}$ & 34 \\
\hline Média & 4 & 0,6 & 5 & & 18 & 53 & 47 & & 19 & 67 & 12 & \\
\hline \multicolumn{2}{|c|}{ C.V. $\%$} & \multicolumn{3}{|l|}{1,61} & \multicolumn{4}{|c|}{3,76} & \multicolumn{4}{|c|}{5,07} \\
\hline & \multicolumn{4}{|c|}{ Curvularia spp. } & \multicolumn{4}{|c|}{ Drechslera spp. } & \multicolumn{4}{|c|}{ Fusarium spp. } \\
\hline $\mathrm{P}(\mathrm{h})$ & $\mathrm{J}$ & $\mathrm{F}$ & M & Média & $\mathrm{J}$ & $\mathrm{F}$ & M & Média & $\mathrm{J}$ & $\mathrm{F}$ & $\mathrm{M}$ & Média \\
\hline 24 & $16 \mathrm{Abc}$ & $19 \mathrm{Aa}$ & $13 \mathrm{Aa}$ & a 16 & 0 & 1 & 0 & $0,4 \mathrm{a}$ & $8 \mathrm{Ba}$ & $39 \mathrm{Aa}$ & $4 \mathrm{Ba}$ & 15 \\
\hline 48 & $10 \mathrm{Ac}$ & $5 \mathrm{Ab}$ & $5 \mathrm{Aa}$ & 6 & 2 & 0 & 0 & $0,8 \mathrm{a}$ & $3 \mathrm{Aa}$ & $8 \mathrm{Aa}$ & $1 \mathrm{Aa}$ & 4 \\
\hline 72 & $20 \mathrm{Ab}$ & $2 \mathrm{Bb}$ & $8 \mathrm{Ba}$ & a 10 & 1 & 1 & 0 & $0,8 \mathrm{a}$ & $4 \mathrm{Ba}$ & $18 \mathrm{Aa}$ & $1 \mathrm{Ba}$ & 8 \\
\hline 96 & $34 \mathrm{Aa}$ & $1 \mathrm{Bb}$ & $5 \mathrm{Ba}$ & a 13 & 0 & 0 & 0 & $0 \mathrm{a}$ & $2 \mathrm{Ba}$ & $50 \mathrm{Aa}$ & $6 \mathrm{Ba}$ & 18 \\
\hline Média & 19 & 7 & 8 & & $0,8 \mathrm{~A}$ & $0,6 \mathrm{~A}$ & $0,1 \mathrm{~A}$ & & 4 & 28 & 3 & \\
\hline C.V.\% & & 3,22 & & & & 0,95 & & & & 4,39 & & \\
\hline
\end{tabular}

${ }^{*}$ Médias seguidas pela mesma letra maiúscula na coluna e minúscula na linha não diferem entre si pelo teste de Tukey, em nível de 5\% de probabilidade. J: janeiro; F: fevereiro; M: março. h: tempo de exposição em horas.

A correlação entre a incidência de Aspergillus spp. e Penicillium spp. e a porcentagem de sementes mortas e germinação (Tabela 6) possibilitou verificar que o período de 24h de exposição à condições adversas do envelhecimento acelerado não apresentou dados que se correlacionassem significativamente, pois nenhum dos fungos afetou a germinação e o número de sementes mortas. Já nos tempos de 48 e $72 \mathrm{~h}$, as sementes colhidas em março apresentaram maiores coeficientes de correlação entre porcentagem de ocorrência de Aspergillus spp. e Penicilium spp. e o número de sementes mortas, com $1 \%$ de significância, possibilitando verificar que a maior incidência desses patógenos resultou em um conseqüente aumento na porcentagem de sementes mortas. Estudos realizados por Muniz et al. (2004), com sementes de melão, demonstraram coeficientes de correlação significativos entre a incidência de Aspergillus spp. e a porcentagem de sementes mortas, confirmando que este é um dos principais fungos causadores de podridão em sementes.

Além disso, no período de $72 \mathrm{~h}$ também ocorreu correlação significativa negativa entre germinação e ocorrência dos patógenos, demonstrando a influência prejudicial dos fungos na germinação. Resultado semelhante pode ser observado quando as sementes foram expostas à $96 \mathrm{~h}$ de envelhecimento, ou seja, quanto maior a porcentagem de fungos associados com as mesmas, menor a porcentagem de sementes germinadas.

No geral, Aspergillus spp. causou maior prejuízo na germinação das sementes de zínia do que Penicillium spp. (Tabela 6). Segundo Silva e Silva (2000), as condições do envelhecimento acelerado favorecem o desenvolvimento de microrganismos, destacando a tendência de domínio de Aspergillus spp. sobre os demais fungos presentes. 
TABELA 6. Coeficientes de correlação simples (r) entre as variáveis porcentagem de germinação (G) e de sementes mortas (SM) e a incidência dos fungos Aspergillus spp. e Penicillium spp. em sementes de Zínia colhidas em janeiro (J), fevereiro (F) e março (M) de 2005 e submetidas a 24, 48, 72 e 96 horas de envelhecimento acelerado.

\begin{tabular}{|c|c|c|c|c|c|c|c|c|c|c|c|c|}
\hline \multicolumn{7}{|c|}{24 horas } & \multicolumn{6}{|c|}{48 horas } \\
\hline \multicolumn{4}{|c|}{ Aspergillus spp. } & \multicolumn{3}{|c|}{ Penicillium spp. } & \multicolumn{3}{|c|}{ Aspergillus spp. } & \multicolumn{3}{|c|}{ Penicillium spp. } \\
\hline & $\mathrm{J}$ & $\mathrm{F}$ & $\mathrm{M}$ & $\mathrm{J}$ & $\mathrm{F}$ & $\mathrm{M}$ & $\mathrm{J}$ & $\mathrm{F}$ & $\mathrm{M}$ & $\mathrm{J}$ & $\mathrm{F}$ & $\mathrm{M}$ \\
\hline $\mathrm{G}$ & 0,00 & 0,20 & 0,21 & $-0,07$ & 0,11 & 0,19 & 0,52 & $-0,22$ & $-0,33$ & $-0,63^{*}$ & 0,02 & $-0,52$ \\
\hline SM & 0,00 & 0,44 & 0,36 & 0,00 & 0,46 & $-0,09$ & 0,02 & $-0,29$ & $0,70^{*}$ & $-0,34$ & 0,22 & $0,65^{*}$ \\
\hline \multicolumn{7}{|c|}{72 horas } & \multicolumn{6}{|c|}{96 horas } \\
\hline & \multicolumn{3}{|c|}{ Aspergillus spp. } & \multicolumn{3}{|c|}{ Penicillium spp. } & \multicolumn{3}{|c|}{ Aspergillus spp. } & \multicolumn{3}{|c|}{ Penicillium spp. } \\
\hline & $\mathrm{J}$ & $\mathrm{F}$ & M & $\mathrm{J}$ & $\mathrm{F}$ & M & $\mathrm{J}$ & $\mathrm{F}$ & M & $\mathrm{J}$ & $\mathrm{F}$ & M \\
\hline $\mathrm{G}$ & $-0,72 *$ & $-0,22$ & $-0,75^{*}$ & $-0,25$ & $-0,58$ & $0,66^{*}$ & $-0,26$ & $-0,69^{*}$ & $-0,84 *$ & $-0,64^{*}$ & $-0,51$ & $-0,47$ \\
\hline SM & 0,49 & $-0,11$ & $0,85^{*}$ & $-0,13$ & $-0,36$ & $0,75^{*}$ & 0,48 & 0,37 & 0,23 & $0,76^{*}$ & 0,40 & 0,26 \\
\hline
\end{tabular}

$*=$ significativo a $1 \%$ de probabilidade.

Portanto, a expressão do potencial fisiológico e da sanidade das sementes depende não só do histórico de produção, mas, principalmente, das condições do ambiente em que as sementes foram expostas.

\section{CONCLUSÕES}

O teste de envelhecimento acelerado, conduzido a $40^{\circ} \mathrm{C}$ por 72 e 96 horas, é eficiente para diferenciação da qualidade fisiológica das sementes de Zinnia elegans Jacq., colhidas em diferentes épocas.

O fungo Aspergillus spp., associado às sementes, influencia negativamente na germinação.

\section{REFERÊNCIAS}

BARNETT, H.L.; HUNTER, B.B. Illustrated genera of imperfect fungi. St Paul, Minnesota: APS Press, 1998. 218p.

BRASIL. Ministério da Agricultura e Reforma Agrária. Regras para análise de sementes. Brasília: SNDA/DNDV/ CLAV, 1992. 365p.

BARROS, D. I.; NUNES, H.V.; DIAS, D.C.F.S.; BHERING, M.C. Comparação entre testes de vigor para avaliação da qualidade fisiológica de sementes de tomate. Revista Brasileira de Sementes, Brasília, v.24, n.2, p.12-16, 2002.

CARDOSO, A.I.I. Produção e qualidade de sementes de abobrinha "Piramoita" em resposta à quantidade de pólen.
Bragantia, Campinas, v.62, n.1, p.47-52, 2003.

CARVALhO, N.M. de; NAKAGAWA, J. Sementes: Ciência, Tecnologia e Produção. Jaboticabal: FUNEP, 2000. 588p.

CHOUDHURY, M. M. Testes de sanidade de sementes de caupi. In: SOAVE, Y. \& WETZEL, M. M. V. da S. Patologia de sementes. Campinas: Fundação Cargill, 1987. p.371-385.

GEMTCHÚJNICOV, I.D. Manual de Taxonomia Vegetal: plantas de interesse econômico. São Paulo: Editora Agronômica Ceres, 1976. 368p.

GUIMARÃES, T.G.; OLIVEIRA, D.A.; MANTOVANIALVARENGA, E.; GROSSI, J.A.S. Maturação fisiológica de sementes de zínia (Zinnia elegans Jacq.). Revista Brasileira de Sementes, Brasília, v.20, n.1, p. 7-11, 1998.

HALLOIN, J. M.. Postharvest infection of cotton seed by Rhizopus arrhizus, Aspergillus niger and Aspergillus flavus. Phytopathology, St. Paul, v. 65, n.11, p. 1229 - 1232, 1975.

JUNQUEIRA, A.H.; PEETZ, MS. Os pólos de produção de flores e plantas ornamentais do Brasil: uma análise do potencial exportador. Revista Brasileira de Horticultura Ornamental, Campinas, v.8, n.1/12, p. 25-47, 2002.

LOPES, J. C.; DIAS, P. C.; PEREIRA, M. D. Maturação fisiológica de sementes de quaresmeira. Pesquisa Agropecuária Brasileira, Brasilia, v.40, n.8, p. 811-816, 2005.

LORENZI, H.; SOUZA, H.M. Plantas ornamentais no Brasil: 
arbustivas, herbáceas e trepadeiras, 2ed. São Paulo: Instituto Plantarum de Estudos da Flora. 1999. 1.088p.

MACHADO, J.C. Patologia de sementes: fundamentos e aplicações. Lavras: ESAL/FAEPE, 1988. 107p.

MARCOS FILHO, J. Teste de envelhecimento acelerado. In: KRZYZANOWSKI, F. C.; VIEIRA, R.D.; FRANÇA NETO, J.B. (Ed.). Vigor de sementes: conceitos e testes. Londrina: ABRATES, 1999. p.3.1-3.24.

MARITAN, A.C.; GASPAR, J.O.; CAMARGO, L.E.A. Identificação e caracterização de um potyvírus isolado de Zinnia elegans. Fitopatologia Brasileira, Brasília, v.29, n.1, p. 24-29, 2004.

MENTEN, J.O. Patógenos em sementes: detecção, danos e controle químico. São Paulo: CibaAgro. 1995. 231p.

MIRANDA, D.M.; NOVEMBRE, A.D.L.C.; CHAMMA, H.M.C.P. Avaliação do potencial fisiológico de sementes de sorgo pelo teste de envelhecimento acelerado. Revista Brasileira de Sementes, Brasília, v.23, n.1, p.226-231, 2001.

MUNIZ, M. F. B.; GONÇALVES, N.; GARCIA, D. C.; KULCZYNSKI, S. M. Comparação entre métodos para avaliação da qualidade fisiológica e sanitária de sementes de melão. Revista Brasileira de Sementes, Brasília, v.26, n.2, p.144-149, 2004.

NAKAGAWA, J. Teste de vigor baseado na avaliação das plântulas. In: KRZYZANOWSKI, F.C.; VIEIRA, R.D.;
FRANÇA NETO, J.B. (Ed.) Vigor de sementes: conceitos e testes. Londrina: ABRATES, 1999. p.2-1 - 2-21.

NASCIMENTO, W. M.; BARROS, de. B. G. C.; PESSOA, H. de. V.S.B. Teste de envelhecimento acelerado em sementes de tomate. Revista Brasileira de Sementes, Brasília, v.15, n.2, p.251-253, 1993.

NEERGAARD, P. Seed pathology. London: Mc Millan Press 2v. 1979. 1191p.

POPINIGIS, F. Fisiologia da semente. Brasília: AGIPLAN, 1985. $289 \mathrm{p}$.

SADER, R.; SILVEIRA, M. M. Maturação fisiológica de sementes de girassol cv. IAC-Anhandy. Revista Brasileira de Sementes, Brasília, v. 10, n.3, p. 9-18, 1988.

SILVA, M. A. D.; SILVA, W. R. Comportamento de fungos e de sementes de feijoeiro durante o teste de envelhecimento artificial. Pesquisa Agropecuária Brasileira, Brasília, v.35, n3, p. 599-608, 2000.

SMIDERLE, O. J.; PEREIRA, P. R. V. da S. Épocas de colheita e qualidade fisiológica das sementes de arroz irrigado cultivar BRS 7 TAIM, em Roraima. Revista Brasileira de Sementes, Brasília, v. 30, n.1, p.74-80, 2008.

TORRES, A.M. Taxonomy of Zinnia. Brittonia, New York. v.15, p. 1-25, 1963.

ZONTA, E.P.; MACHADO, A.A. Sistema de análise estatística para microcomputadores - SANEST. Pelotas: UFPel, 1986. 